\title{
MicroRNA preparations from individual monogenean Gyrodactylus salaris-a comparison of six commercially available totalRNA extraction kits
}

\author{
Bastian Fromm*, Philip David Harris and Lutz Bachmann
}

\begin{abstract}
Background: Describing and evaluating miRNA inventories with Next Generation Sequencing is a goal of scientists from a wide range of fields. It requires high purity, high quality, and high yield RNA extractions that do not only contain abundant ribosomal RNAs but are also enriched in miRNAs. Here we compare 6 disparate and commercially available totalRNA extraction kits for their suitability for miRNA-preparations from Gyrodactylus salaris, an important but small (500 $\mu \mathrm{m}$ in length) monogenean pathogen of Norwegian Atlantic salmon (Salmo salar).

Findings: We evaluated 1 salt precipitation method (MasterPure ${ }^{\mathrm{TM}}$ Complete RNA Purification Kit, Epicentre), 2 Phenol based extraction methods (mirVana Kit, Ambion, and Trizol Plus Kit, Invitrogen), 1 paramagnetic bead extraction method (RNA Tissue kit, GeneMole) and 2 purification methods based on spin column chromatography using a proprietary resin as separation matrix (Phenol-free Total RNA Purification Kit, Amresco, and ZR MicroPrep Kit, Zymo Research). The quality of the extractions from 1, 10 and 100 individuals, respectively, was assessed in terms of totalRNA yield, RNA integrity, and smallRNA and miRNA yield. The 6 RNA extraction methods yielded considerably different total RNA extracts, with striking differences in low molecular weight RNA yield. The Phenolfree Total RNA Purification Kit (Amresco) showed the highest totalRNA yield, but the best miRNA/totalRNA ratio was obtained with the ZR MicroPrep Kit (Zymo Research). It was not possible to extract electrophoretically detectable miRNAs from Gyrodactylus salaris with the RNA Tissue Kit (GeneMole) or the Trizol Plus Kit (Invitrogen).

Conclusions: We present an optimized extraction protocol for single and small numbers of Gyrodactylus salaris from infected Atlantic salmon that delivers a totalRNA yield suitable for downstream next generation sequencing analyses of miRNA. Two of the six tested totalRNA kits/methods were not suitable for the extraction of miRNAs from Gyrodactylus salaris.
\end{abstract}

\section{Background}

MicroRNAs (miRNAs) are key regulators of many biological processes in eukaryotes [1]. Besides their investigation through bioinformatical analyses of whole genomes, research focuses on analyses of miRNAs from whole organisms, specific tissues, and/or developmental stages without a fully sequenced and annotated genome at hand [2].

MiRNAs are single-stranded, 22 nucleotide long, noncoding transcripts derived from different genomeencoded hairpin precursors, and regulate gene expression by various mechanisms [3]. First described from

\footnotetext{
* Correspondence: bastian.fromm@nhm.uio.no

Natural History Museum, University of Oslo, PO Box 1172 Blindern, 0318 Oslo, Norway
}

Caenorhabditis elegans [4] they represent the most recently discovered gene regulators, involved in a broad variety of biological processes including cell proliferation and metabolism [5], developmental timing [6], cell death [7], haematopoiesis [8], neuron development [9], tumorigenesis [10], DNA methylation and chromatin modification [11], and as immune defense against viruses [12]. In evolutionary terms miRNAs are unusual in that they are continuously added to, highly conserved, and rarely lost from metazoan genomes $[13,14]$. Clearly they are under strong selection, and may therefore represent candidate phylogenetic markers. It may even be possible to reconstruct the miRNA complement of the last common ancestor of all Metazoa [15-17]. 
Several methods for isolation of totalRNA have been developed, with the focus primarily on high molecular weight RNAs [18]. Commercially available totalRNA kits are affordable, fast, and suitable for RNA extraction from a broad spectrum of samples. Most manufacturers promote their products as suitable for extraction of totalRNA, but frequently it is unclear whether they are equally suitable for smallRNA species, and especially for miRNAs. For extractions of these molecules not only is overall RNA quality and integrity an issue, but also due to their low abundance, yield is of high importance. This is a particular issue if only limited material is available, as in the case of, for example, micro-dissected tissue samples or small invertebrates.

The current study focuses on the ectoparasitic platyhelminth Gyrodactylus salaris (Monogenea, Gyrodactylidae). This parasite is responsible for a major epidemic disease of wild salmon in Norway and Russia [19], but gyrodactylids are widespread on teleost fishes and several cause disease in aquaculture $[19,20]$. Apart from this applied importance, an understanding of the miRNA complement of gyrodactylids will contribute to our understanding of platyhelminth, and early metazoan evolution. However, the individual parasites are only $500 \mu \mathrm{m}$ in length, presenting a challenge for RNA extraction methodologies. In this study we tested 6 commercially available totalRNA extraction kits for their performance when using as few as 1, 10 and 100 Gyrodactylus salaris individuals respectively. Particular emphasis was placed on assessing (i) total RNA yield (ii) RNA integrity (iii) smallRNA yield, and (iv) miRNA yield.

\section{Methods}

\section{Gyrodactylus salaris culture}

Gyrodactylus salaris were maintained on Atlantic salmon (Salmo salar) parr in 500 liter tanks in charcoal-filtered and dechlorinated, continuously running Oslo tap water at $5-6^{\circ} \mathrm{C}$. Fish were fed daily on pellet feed (Ewos) and maintained under continuous dim illumination [21].

\section{Sampling of Gyrodactylus salaris specimen for totalRNA analyses}

Single infected fish were anaesthetised using $0.1 \%$ chlorbutanol and killed by pithing, taking care to avoid contamination with blood. Fins were cut and stored in $-20^{\circ} \mathrm{C}$ ethanol in $2 \mathrm{ml}$ RNAse-free DNA loBind tubes (Eppendorf) until further processing. Using a binocular microscope Gyrodactylus were removed from fins using a mounted needle, taking care to avoid contamination with fish tissue. Individual parasites were rinsed with ethanol at $-20^{\circ} \mathrm{C}$ to further remove contaminating fish mucus or epithelial cells. For RNA extraction groups of 1,10 , and 100 G. salaris individuals were pooled in $10 \mu \mathrm{l}$ of $-20^{\circ} \mathrm{C}$ ethanol and immediately processed.

\section{Ribonucleic acid extraction}

Six commercially available kits were compared: MasterPure $^{\text {TM }}$ Complete RNA Purification Kit (Epicentre, EPI), Molestrips totalRNA basic for the GeneMole extraction robot (GeneMole, GM), Phenol-free Total RNA Purification Kit (Amresco, AMR), ZR RNA MicroPrep kit (Zymo Research, ZR), mirVana Kit (Ambion, AMB), and the Trizol Plus Kit (Invitrogen, INV).

The selected kits represent four different extraction strategies for total RNA: rapid desalting and precipitation (EPI), solid phase extraction on silica-coated magnetic beads (GM), solid phase extraction on filter membranes (ZR, AMR) and sequential organic and solid phase extractions (AMB and INV). EPI utilises gentle lysis in a SDS-containing buffer with proteinase $\mathrm{K}$ whereas GM, AMR, ZR, and AMB kits all employ guanidinium thiocyanate-(GTC) containing chaotropic buffers for the lysis of the tissue/cells and the simultaneous inactivation of RNAses. The INV kit uses Trizol, a GTC-containing chaotropic lysis-buffer premixed with phenol. The EPI kit consecutively precipitates proteins and nucleic acids from the sample, and uses RNAse free DNAse for the subsequent RNA purification. Following the protocols of the GM, AMR, and ZR kits the samples in the lysis buffer (LB) are directly loaded onto the respective binding matrix, whereas the AMB and the INV kits include an acid-phenol-chloroform extraction step prior to the binding of the RNA to a silica-based glass-fiber filter membrane in a spin cartridge. Under high concentrations of salt the RNA binds to the silica-matrices whereas DNA and Proteins flow through the column. The bound RNA is eventually washed from the filters/beads and collected (Table 1).

For all extractions we followed kit protocols with the minor modification that all extracted RNA was eluted or resuspended in $100 \mu \mathrm{l}$ RNAse-free Water (Ambion). The AMB kit protocol offers also a microRNAs only option but this was not followed as the totalRNA was also in the focus of this study and would have been lost. For the ZR Kit the quantitative smallRNA recovery option was used which should deliver smallRNA enriched total RNA with reasonable high mass RNA as well.

All kits were assessed for extractions from 1, 10, and 100 G. salaris individuals, respectively. The obtained RNA extracts were heat-denatured for $2 \mathrm{~min}$ at $70^{\circ} \mathrm{C}$ and immediately stored in aliquots in $0.5 \mu \mathrm{l}$ RNAse-free DNA loBind tubes (Eppendorf) at $-80^{\circ} \mathrm{C}$ until further use.

\section{Determination of RNA concentrations and RNA integrity}

Quantification of totalRNA yield and assessment of integrity was done with the Experion (Bio-Rad laboratories) and 2100 Bioanalyzer (Agilent systems using the Experion RNA HighSense Analysis Kit and the Agilent RNA 6000 Pico Kit respectively. Both systems make use 
Table 1 Summary of the extraction methods and their chemical principles

\begin{tabular}{lllll}
\hline manufacturer & totalRNA Extraction Kit & lysis & extraction $\mathbf{1}$ & extraction 2 \\
\hline Ambion & mirVana & & organic solvents & $\frac{\text { acid Phenol }}{\text { Trizol }}$ solid-phase \\
Invitrogen & Trizol Plus & Guanidinium-thiocyanat based & & $\frac{\text { beads }}{\text { GeneMole }}$ \\
Zymo & Molestrips totalRNA basic & & solid-phase & CymoReserach RNA miniPrep \\
Amresco & Phenol-free Total RNA Purification & & & none \\
Epicentre & MasterPure Complete RNA Purification & SDS based & precipitation, DNAse treatment \\
\hline
\end{tabular}

Overview of the chemical principles for the assessed totalRNA extraction methods.

of the micro-fluidic electrophoresis technology for the analysis of biological samples. The Small RNA Kit (Agilent) was used to analyse smallRNA and miRNA content with the 2100 Bioanalyzer (Agilent). The Agilent Small RNA kit offers a fast detection of small RNA with a sensitivity higher than agarose or polyacrylamide gels (for further details see: http://www.genomics.agilent.com/ CollectionSubpage.aspx?PageType=Product\&SubPageTy$\mathrm{pe}=$ ProductDetail\&PageID=1647). RNA Quality Indicators (RQI) and RNA integrity numbers (RIN, $1=$ degraded, $10=$ intact), were derived using the system software. All kits were used following the manufacturers protocols, and quantifications were done in duplicate.

\section{Verification of ribosomal RNA}

To verify that totalRNA preparations contained Gyrodactylus salaris RNA all preparations and Salmo salar RNA controls were reverse transcribed into cDNA using the iScript Select cDNA Synthesis Kit (Bio-Rad) and a temperature profile of $25^{\circ} \mathrm{C}$ for $5 \mathrm{~min}, 42^{\circ} \mathrm{C}$ for $30 \mathrm{~min}$, and $85^{\circ}$ for $5 \mathrm{~min}$. The cDNA was probed for $5 \mathrm{~S}, 18 \mathrm{~S}$, and $28 \mathrm{~S}$ rRNA Gyrodactylus salaris using the specific primer pairs 5Sforward: 5'-TCACTCGGCTCACGTGACGA-3', 5Sreverse: 5'-GCCCTTAGCCGCCATTTGCG-3'; 18Sforward: 5'-TGGTTAAACCGCAAACGGCT-3', 18Sreverse: 5'-GT CGTCTGGCAACGGTCCAT-3'; 28Sforward: 5'-CCCAGCACCGAAGCCTACGC-3', 28Sreverse: 5'-AAACCGCT TCGGCCTCCACC-3'. PCR primers were designed from GenBank entries Z72477, Z26942, and AJ542394. The PCR amplification was done using the AmpliTaq Gold ${ }^{\mathbb{B}}$ Fast PCR Master Mix (Applied Biosystems) and a protocol consisting of 30 cycles with $94^{\circ} \mathrm{C}$ for $30 \mathrm{~s}, 60^{\circ} \mathrm{C}$ for $40 \mathrm{~s}$, and $72^{\circ} \mathrm{C}$ for 60 s. Only Gyrodactylus salaris preparations yielded positive PCR products that were verified by DNA sequencing, for control preparations from uninfected fish no PCR products were obtained (not shown).

\section{Results and discussion}

Total RNA yield and integrity

The totalRNA yield and integrity of the extractions were compared. The AMR kit was best for extracting high quality totalRNA (RQI, RIN = 10) from single Gyrodactylus salaris individuals with a yield of $15.4 \mathrm{ng}$ (Figure 1); the other kits yielded less totalRNA and/or total RNA of lower quality (RQI, RIN $<10$ ). The totalRNA yields ranged between $2.8 \mathrm{ng}$ and $25.8 \mathrm{ng}$ per individual. The respective yields for 10 individuals ranged between 19.0 ng and 158.4 ng, and between $212.8 \mathrm{ng}$ and $190.8 \mu \mathrm{g}$ for 100 individuals (Table 2 and Figure 2). The overall average yield was $9.7 \mathrm{ng} \pm 6$ /individual.

The yield differences between the kits were highest for extractions from just 1 individual. This may reflect different developmental stages of the respective animals, but may also be due to uncertain estimates due to the sensitivity limitations of the electrophoretic systems. RNA concentrations of less than $50 \mathrm{pg} / \mu \mathrm{l}$ and $100 \mathrm{pg} / \mu \mathrm{l}$ (corresponding to $5 \mathrm{ng}$ and $10 \mathrm{ng}$ in $100 \mu \mathrm{l}$ ) are below the sensitivity limit of the 2100 Bioanalyzer and Experion systems, respectively. Such effects are expected to be less important for the extractions of 10 and 100 individuals.

In general, the quality of totalRNA extractions from single worms was low (RQI/RIN $5.3 \pm 3.1$ ) but was much better for $10(7.5 \pm 3.4)$ and best for $100(8.1 \pm 2.4)$ individuals. All preparations with the ZR and the EPI kits

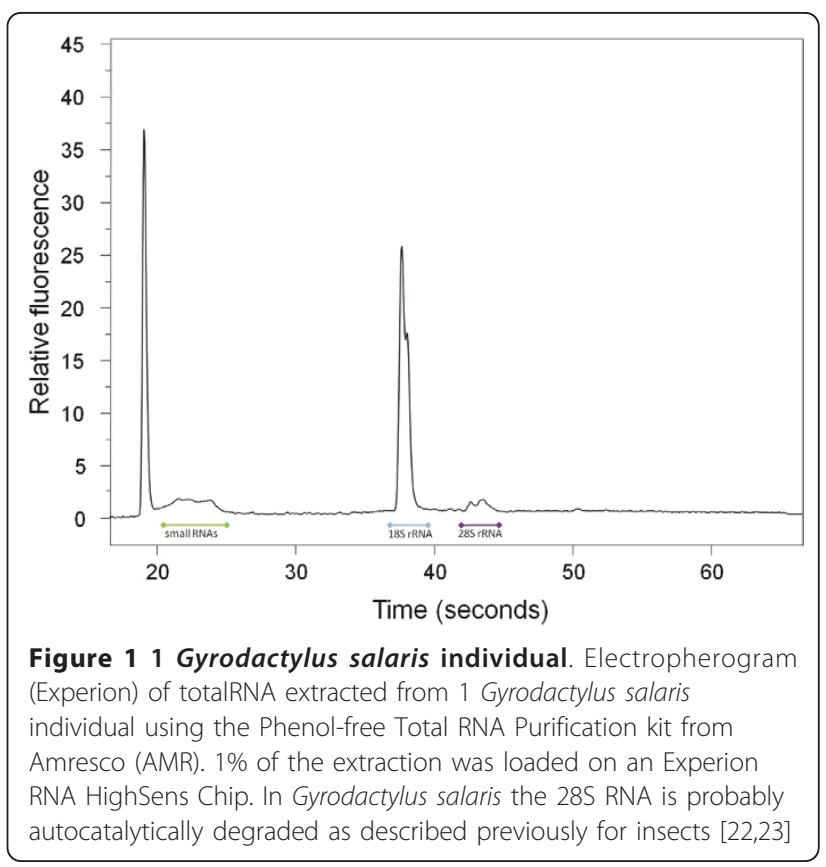


Table 2 totaIRNA yield in $\mathrm{ng}$

\begin{tabular}{|c|c|c|c|c|c|c|c|c|}
\hline \multirow{2}{*}{$\begin{array}{l}\text { Number of Gyrodactylus } \\
\text { salaris individuals } \\
\text { totalRNA Extraction Kit }\end{array}$} & \multicolumn{2}{|c|}{1} & \multicolumn{2}{|c|}{10} & \multicolumn{2}{|c|}{100} & \multicolumn{2}{|c|}{ average per individual } \\
\hline & yield SD & Quality SD & yield SD & quality SD & yield SD & quality SD & yield SD & quality SD \\
\hline Trizol Plus & $2.8 \pm 0.8$ & $2.6 \pm 1.1$ & $19.0 \pm 2.2$ & $9.6 \pm 0.4$ & $212.8 \pm 50.6$ & $9.9 \pm 0.1$ & $2.3 \pm 0.4$ & $7.4 \pm 4.1$ \\
\hline Molestrips totalRNA basic & $5.0 \pm 0.9$ & $8.3 \pm 0.2$ & $75.7 \pm 14.2$ & $10.0 \pm 0.1$ & $562.7 \pm 245.2$ & $9.4 \pm 1.3$ & $6.1 \pm 1.4$ & $9.2 \pm 0.9$ \\
\hline mirVana & $7.8 \pm 0.6$ & $6.7 \pm 1.3$ & $91.1 \pm 11.6$ & $9.4 \pm 0.9$ & $410.9 \pm 53.8$ & $9.7 \pm 0.5$ & $7.0 \pm 2.6$ & $8.6 \pm 1.6$ \\
\hline ZymoResearch RNA miniprep & $20.7 \pm 2.4$ & $2.7 \pm 0.3$ & $74.0 \pm 5.2$ & $3.0 \pm 0.1$ & $576.3 \pm 124.9$ & $4.0 \pm 0.5$ & $11.3 \pm 8.2$ & $3.2 \pm 0.7$ \\
\hline $\begin{array}{l}\text { Phenol-free Total RNA } \\
\text { Purification }\end{array}$ & $15.4 \pm 6.1$ & $9.2 \pm 1.2$ & $142.0 \pm 27.8$ & $9.9 \pm 0.2$ & $1908.5 \pm 500.6$ & $9.5 \pm 0.9$ & $16.2 \pm 2.5$ & $9.5 \pm 0.4$ \\
\hline $\begin{array}{l}\text { MasterPure Complete RNA } \\
\text { Purification }\end{array}$ & $25.8 \pm 4.3$ & $2.3 \pm 0.2$ & $158.4 \pm 25.9$ & $3.2 \pm 0.5$ & $505.0 \pm 59.7$ & $6.2 \pm 0.7$ & $15.6 \pm 10.4$ & $3.9 \pm 2.0$ \\
\hline average absolute & $12.9 \pm 9.2$ & $5.3 \pm 3.1$ & $93.4 \pm 50.6$ & $7.5 \pm 3.4$ & $696.0 \pm 608.9$ & $8.1 \pm 2.4$ & $9.7 \pm 5.6$ & $7.0 \pm 2.7$ \\
\hline average per individual & $12.9 \pm 9.2$ & $5.3 \pm 3.1$ & $9.3 \pm 5.1$ & $7.5 \pm 3.4$ & $7.0 \pm 6.1$ & $8.1 \pm 2.4$ & & \\
\hline
\end{tabular}

TotalRNA yield and quality of all assessed totalRNA extraction kits for 1, 10 and 100 Gyrodactylus salaris specimens. RNA-quality is expressed as RQI/RIN values determined by the software following the Experion and 2100 Bioanalyzer electrophoresis systems respectively. The global average over all methods and sample sizes is depicted in bold.

SD-standard deviation

had relatively low quality indices (2.3-6.2), an indication of increased levels of degradation. The rather poor baseline in the electropherogram shows that, particularly in the EPI preparation, the RNA integrity had suffered from a relatively long extraction time including DNAse treatment. For the ZR kit, however, the low RQI/RIN values seem to have a different explanation. The protocol efficiently extracts small RNAs and the high molecular weight RNAs contribute comparatively less to the totalRNA. Although there is no noise indicating RNA degradation (Figure 3), the distribution of RNA seems to have been misinterpreted by the analysis algorithm as an indication of poor quality.

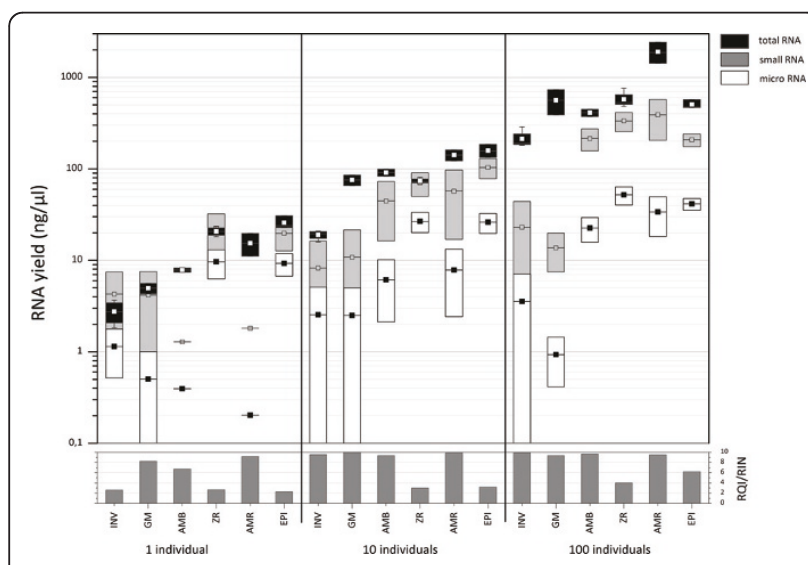

Figure 2 Boxplot summary of all data. Semilogarithmic boxplot breakdown of RNA yield for totalRNA, smallRNA and microRNA of all assessed totalRNA extraction kits for 1,10 and 100 Gyrodacty/us salaris specimens. RQI/RIN values of the totalRNA as determined by the instrument software following the Experion and 2100 Bioanalyzer electrophoresis systems are depicted at the bottom. Boxes indicate the $25-75 \%$ intervals, squares the mean, and whiskers the standard deviation, where this lies outside the $25-75 \%$ interval.
Interestingly the assessed RNA extraction kits did not only show differences in yield per individual, they also differed significantly in yield per individual between the extractions using 1,10 , and 100 individuals, respectively. This is unlikely to be due to exceeding the extraction limits of the respective kits, and it is also unlikely - at least for the EPI kit, as it is a precipitation kit only-to be a consequence of the binding capacity of the corresponding binding matrix.

The electrophoretic profile of all Gyrodactylus preparations with a major $18 \mathrm{~S}$ rRNA peak as the most

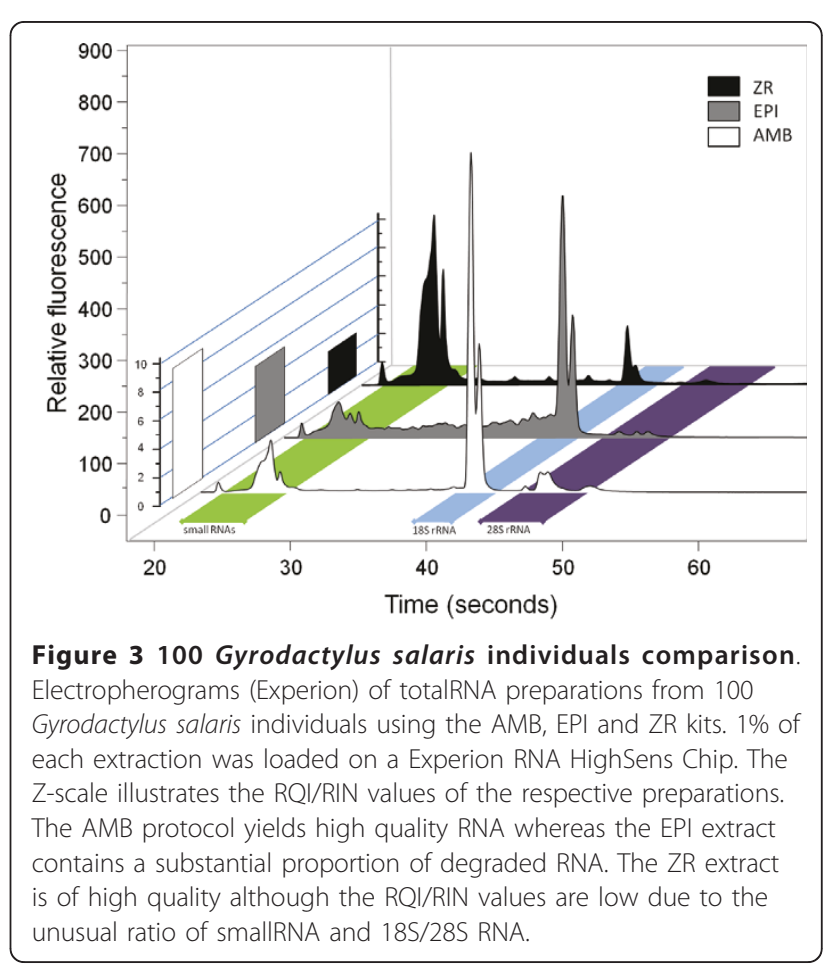


prominent signal was different from the "usual" totalRNA preparation profile with a high $28 \mathrm{~S}$ rRNA signal, a less prominent $18 \mathrm{~S}$ rRNA signal, and a rather low signal from the smallRNAs. A very similar pattern has been reported from insect RNA preparations. For several insects it has been shown to be a result of heatand chemical-dissociable $28 \mathrm{~S}$ rRNA species, which autocatalytically degrade into two $18 \mathrm{~S}$ rRNA pieces [22,23]. Similar processes may also affect the $28 \mathrm{~S}$ rRNA of Gyrodactylus. In our study this peculiarity of Gyrodactylus $28 \mathrm{~S}$ rRNA allows us to detect sample contamination with fish RNA. As the Atlantic salmon 28S rRNA does not show this property (Figure 4), a very prominent $28 \mathrm{~S}$ rRNA peak would point to sample contamination. No such contamination has been observed in any Gyrodactylus preparation.

\section{Small and microRNA yield}

As expected the yield of smallRNA of the assessed kits varied as the totalRNA yield: for 1 individual the values ranged between $1.3 \mathrm{ng}$ and $22.3 \mathrm{ng}$, between 8.2 $\mathrm{ng}$ and 103.4 ng for 10, and between $13.6 \mathrm{ng}$ and 389.3 ng for 100 individuals (see Table 3). The overall average yield of smallRNAs was $5.3 \mathrm{ng} /$ individual. The smallRNA fraction includes the microRNAs, and therefore it was no surprise that the microRNA yield differed almost proportionally to the smallRNA yield. The respective values ranged between $0.2 \mathrm{ng}$ and $9.6 \mathrm{ng}$ for 1, between $2.5 \mathrm{ng}$ and $26.6 \mathrm{ng}$ for 10, and between 0.9 $\mathrm{ng}$ and $51.6 \mathrm{ng}$ for 100 individuals (see Figure 2). The overall average yield of microRNAs was $1.7 \mathrm{ng} /$ individual. The best microRNA to smallRNA ratio (with the highest absolute microRNA value of $51.6 \mathrm{ng}$ ) was

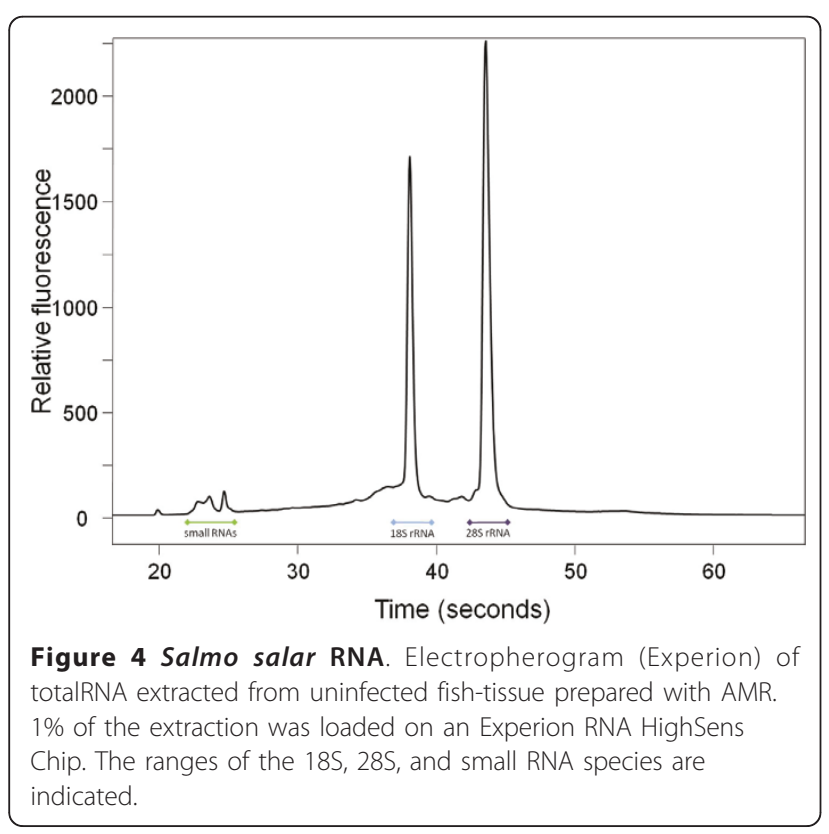

achieved with the ZR kit for 100 individuals. In contrast, only very small amounts of either smallRNAs or microRNAs could be detected in extracts from the GM and the INV kits.

Meaningful assessment of the yield of smallRNAs depended on the quality of the sample, as an electropherogram of degraded RNA may resemble that of samples with a high smallRNA content. Given the low RQI/ RIN values we consider the relatively large smallRNA fraction obtained with the EPI kit as mainly consisting of degraded high molecular weight RNA.

Summarizing the data gathered in this study an average Gyrodactylus salaris individual yields $9.7 \mathrm{ng}$ totalRNA out of which $5.3 \mathrm{ng}$ are small RNAs, including 1.7 ng microRNAs.

\section{Conclusions}

We have demonstrated successful extraction of high quality RNA from a single Gyrodactylus salaris individual, a small monogenean ectoparasite with a body length of $500 \mu \mathrm{m}$ only. Careful choice of totalRNA extraction kit is crucial, and in our comparison, the best results for totalRNA containing smallRNAs were obtained with the Phenol-free Total RNA Purification Kit (Amresco). However the highest amount of miRNAs with a yield of $9.6 \mathrm{ng}$ for one individual could be extracted using the ZR RNA MicroPrep kit (Zymo Research), which is also the only kit that allows elution with a volume as little as $6 \mu \mathrm{l}$, one order of magnitude smaller than for the other kits and recommended as input for some downstream NGS applications $(8 \mu \mathrm{l}$, ScriptMinerTM Small RNA-Seq Library Preparation Kit, Lit. \# $316 \cdot 11 / 2010$, Cambio).

The amount of totalRNA extracted in this study suggests that a sample size of 100 Gyrodactylus salaris individuals is sufficient for a microRNAs sequencing run with an Illumina Genome Analyzer 2 (1-5 $\mu \mathrm{g}$, ScriptMiner ${ }^{\mathrm{TM}}$ Small RNA-Seq Library Preparation Kit, Lit. \# 316 . 11/2010, Cambio), making such genomic and transcriptomic analyses feasible with manageable numbers of these pathogenic fish parasites.

Other totalRNA extractions kits such as the Molestrips totalRNA basic for the GeneMole extraction robot (GeneMole) and the Trizol Plus Kit (Invitrogen) were found to be inappropriate for extracting small RNA and microRNA from rather small samples. This may be of particular interest as kits from the Trizol (Invitrogen) family have frequently been used as the standard extraction method in recent microRNAs studies (e.g., [17]).

The successful extraction of suitable amounts of RNA, especially miRNAs of reasonable quality offers an interesting novel tool for assessing the miRNA complement of single small sized parasites including those of different developmental stages. 
Table 3 small/miRNA yield in ng

\begin{tabular}{|c|c|c|c|c|c|c|c|c|}
\hline $\begin{array}{l}\text { Number of Gyrodactylus salaris } \\
\text { individuals }\end{array}$ & 1 & & 10 & & 10 & & average per & individual \\
\hline totalRNA Extraction Kit & smallRNA SD & miRNA SD & smallRNA SD & miRNA SD & smallRNA SD & miRNA SD & smallRNA SD & miRNA SD \\
\hline Trizol Plus & $4.3 \pm 4.5$ & $1.1 \pm 0.9$ & $8.2 \pm 11.2$ & $2.5 \pm 3.6$ & $22.9 \pm 29.6$ & $3.5 \pm 5.0$ & $1.8 \pm 2.2$ & $0.5 \pm 0.6$ \\
\hline Molestrips totalRNA basic & $4.2 \pm 4.7$ & $0.5 \pm 0.7$ & $10.8 \pm 15.1$ & $2.5 \pm 3.5$ & $13.6 \pm 8.8$ & $0.9 \pm 0.7$ & $1.8 \pm 2.1$ & $0.2 \pm 0.2$ \\
\hline mirVana & $1.3 \pm$ n.a. & $0.4 \pm$ n.a. & $44.4 \pm 39.9$ & $6.1 \pm 5.6$ & $214.2 \pm 81.7$ & $22.4 \pm 9.6$ & $2.6 \pm 1.6$ & $0.4 \pm 0.2$ \\
\hline ZymoResearch RNA miniprep & $22.3 \pm 13.8$ & $9.6 \pm 4.8$ & $70.0 \pm 28.5$ & $26.6 \pm 9.4$ & $333.4 \pm 112.0$ & $51.6 \pm 16.4$ & $10.9 \pm 10.1$ & $4.3 \pm 4.7$ \\
\hline $\begin{array}{l}\text { Phenol-free Total RNA } \\
\text { Purification }\end{array}$ & $1.8 \pm$ n.a. & $0.2 \pm$ n.a. & $56.9 \pm 56.6$ & $7.8 \pm 7.6$ & $389.3 \pm 261.0$ & $33.7 \pm 22.0$ & $3.8 \pm 2.0$ & $0.4 \pm 0.3$ \\
\hline $\begin{array}{l}\text { MasterPure Complete RNA } \\
\text { Purification }\end{array}$ & $19.7 \pm 10.0$ & $9.2 \pm 3.6$ & $103.4 \pm 36.5$ & $25.9 \pm 9.0$ & $207.7 \pm 47.1$ & $41.1 \pm 8.7$ & $10.7 \pm 8.8$ & $4.1 \pm 4.6$ \\
\hline average absolute & $8.9 \pm 9.5$ & $3.5 \pm 4.6$ & $49.0 \pm 36.4$ & $11.9 \pm 11.3$ & $196.9 \pm 154.9$ & $25.6 \pm 20.5$ & $5.3 \pm 4.3$ & $1.7 \pm 2.0$ \\
\hline average per individual & $9.7 \pm 9.2$ & $3.9 \pm 4.4$ & $4.9 \pm 3.6$ & $1.2 \pm 1.1$ & $2.0 \pm 1.5$ & $0.3 \pm 0.2$ & & \\
\hline
\end{tabular}

SmallRNA and microRNA yield of all assessed totalRNA extraction kits for 1, 10 and 100 Gyrodactylus salaris specimen. The global average over all methods and sample sizes is depicted in bold.

SD-standard deviation

\section{Acknowledgements}

The authors thank Morten Skage for offering the opportunity to use the 2100 Bioanalyzer available at the DNA lab of the Centre for Ecological and Evolutionary Synthesis (CEES), University of Oslo, Norway.

\section{Authors' contributions}

BF conceived the study, designed and carried out all the experiments and drafted the manuscript. LB and PDH participated in the design of the study and contributed to drafting the manuscript. All authors have read and approved the final version of the manuscript.

\section{Competing interests}

The authors declare that they have no competing interests.

Received: 11 March 2011 Accepted: 29 June 2011

Published: 29 June 2011

\section{References}

1. Krol J, Loedige I, Filipowicz W: The widespread regulation of microRNA biogenesis, function and decay. Nature Rev Genet 2010, 11:597-610.

2. Xu M-J, Liu Q, Nisbet A, Cai X-Q, Yan C, Lin R-Q, Yuan Z-G, Song H-Q, He X$H$, Zhu X-Q: Identification and characterization of microRNAs in Clonorchis sinensis of human health significance. BMC Genomics 2010, 11:521.

3. Huntzinger $E$, Izaurralde E: Gene silencing by microRNAs: contributions of translational repression and mRNA decay. Nat Rev Genet 2011, 12:99-110.

4. Lee $R$, Feinbaum $R$, Ambros $V$ : The $C$. elegans heterochronic gene lin-4 encodes small RNAs with antisense complementarity to lin-14. Cell 1993, 75:843-854.

5. Brennecke J, Cohen SM: Towards a complete description of the microRNA complement of animal genomes. Genome biology 2003, 4:228.

6. Reinhart B, Slack F, Basson M, Pasquinelli A, Bettinger J, Rougvie A, Horvitz H, Ruvkun G: The 21-nucleotide let-7 RNA regulates developmental timing in Caenorhabditis elegans. Nature 2000, 403:901-906.

7. Baehrecke EH: miRNAs: micro managers of programmed cell death. Curr Biol 2003, 13:R473-475.

8. Chen J, Li WX, Xie D, Peng JR, Ding SW: Viral virulence protein suppresses RNA silencing-mediated defense but upregulates the role of microrna in host gene expression. The Plant cell 2004, 16:1302-1313.

9. Johnston RJ, Hobert O: A microRNA controlling left/right neuronal asymmetry in Caenorhabditis elegans. Nature 2003, 426:845-849.

10. Michael MZ, SM OC, van Holst Pellekaan NG, Young GP, James RJ: Reduced accumulation of specific microRNAs in colorectal neoplasia. Mol Cancer Res 2003, 1:882-891.

11. Bao N, Lye KW, Barton MK: MicroRNA binding sites in Arabidopsis class III HD-ZIP mRNAs are required for methylation of the template chromosome. Developmental cell 2004, 7:653-662.
12. Sarnow $\mathrm{P}$, Jopling $\mathrm{CL}$, Norman $\mathrm{KL}$, Schutz $\mathrm{S}$, Wehner $\mathrm{KA}$ : MicroRNAs: expression, avoidance and subversion by vertebrate viruses. Nat Rev Microbiol 2006, 4:651-659.

13. Sempere LF, Cole CN, McPeek MA, Peterson KJ: The phylogenetic distribution of metazoan microRNAs: insights into evolutionary complexity and constraint. J Exp Zool B Mol Dev Evol 2006, 306:575-588.

14. Heimberg AM, Sempere LF, Moy VN, Donoghue PC, Peterson KJ: MicroRNAs and the advent of vertebrate morphological complexity. Proc Natl Acad Sci USA 2008, 105:2946-2950.

15. Christodoulou F, Raible F, Tomer R, Simakov O, Trachana K, Klaus S, Snyman H, Hannon GJ, Bork P, Arendt D: Ancient animal microRNAs and the evolution of tissue identity. Nature 2010, 463:1084-1088.

16. Sempere LF, Martinez P, Cole C, Baguna J, Peterson KJ: Phylogenetic distribution of microRNAs supports the basal position of acoel flatworms and the polyphyly of Platyhelminthes. Evol Dev 2007, 9:409-415.

17. Wheeler BM, Heimberg AM, Moy VN, Sperling EA, Holstein TW, Heber S, Peterson KJ: The deep evolution of metazoan microRNAs. Evol Dev 2009 11:50-68.

18. Farrell RE: RNA methodologies. Laboratory guide for isolation and characterization. Fourth edition. Academic Press, Burlington MA; 2010.

19. Bakke TA, Cable J, Harris PD: The biology of gyrodactylid monogeneans: the "Russian-doll killers". Adv Parasitol 2007, 64:161-376.

20. Harris PD, Shinn AP, Cable J, Bakke TA: Nominal species of the genus Gyrodactylus von Nordmann 1832 (Monogenea: Gyrodactylidae), with a list of principal host species. Syst Parasitol 2004, 59:1-27.

21. Olstad K, Robertsen G, Bachmann L, Bakke TA: Variation in host preference within Gyrodactylus salaris (Monogenea): an experimental approach. Parasitology 2007, 134:589-597.

22. Ishikawa $H$, Newburgh RW: Studies of the thermal conversion of $28 \mathrm{~S}$ RNA of Galleria mellonella (L.) to an 18 S product. J Mol Biol 1972 , 64:135-144.

23. Jäckle H, Schmidt $\mathrm{O}$ : Characterization of ribosomal RNA from insect eqqs (Euscelis plebejus, Cicadina; Smittia spec., Chironomidae, Diptera). Experientia 1978, 34:1260-1261.

doi:10.1186/1756-0500-4-217

Cite this article as: Fromm et al:: MicroRNA preparations from individual monogenean Gyrodactylus salaris-a comparison of six commercially available totalRNA extraction kits. BMC Research Notes 2011 4:217. 